\title{
Le journalisme citoyen au Mozambique : nature, légitimité et enjeux sociétaux
}

Citizen Jornalism in Mozambique: Nature, legitimacy and societal issues Jornalismo cidadao no Moçambique : natureza, legtimidade e sociais efeitos

\section{Maud Pélissier et César CUMBE}

\section{OpenEdition}

\section{Journals}

Édition électronique

URL : http://journals.openedition.org/ctd/2309

DOI : $10.4000 /$ ctd.2309

ISSN : 2491-1437

Éditeur

Chaire Unesco Pratiques émergentes en technologies et communication pour le développement

Édition imprimée

Date de publication : 12 novembre 2015

Référence électronique

Maud Pélissier et César CUMBE, «Le journalisme citoyen au Mozambique : nature, légitimité et enjeux sociétaux », Communication, technologies et développement [En ligne], 2 | 2015, mis en ligne le 10 juin 2019, consulté le 22 juillet 2020. URL : http://journals.openedition.org/ctd/2309 ; DOI : https://doi.org/ 10.4000/ctd.2309 


\section{BIBLIOTHÈQUES, CONSERVATOIRES, OBSERVATOIRES}

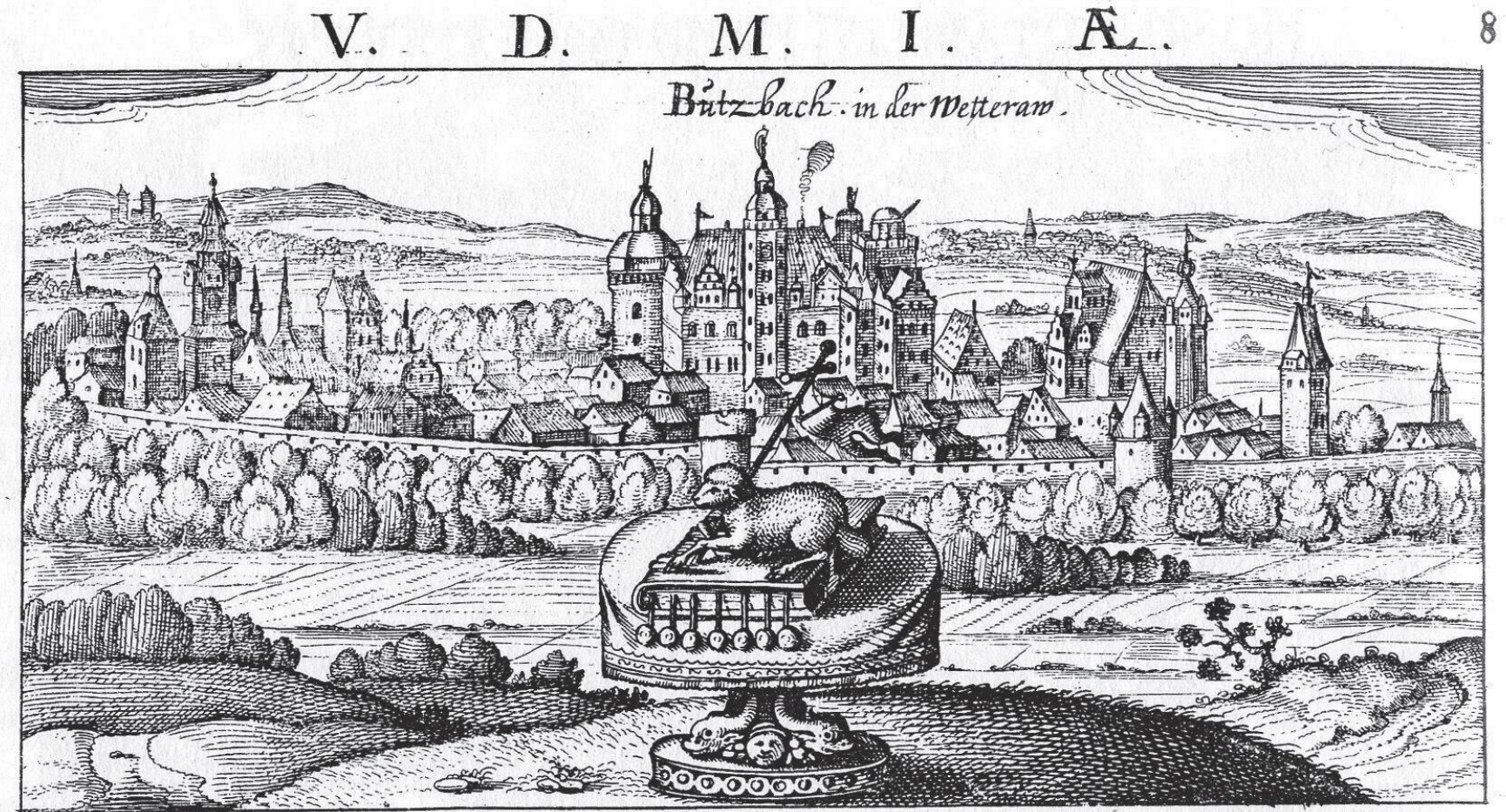

Omnia difpereant, gúoniam periijse necefse est, Verbi üfüs maneat fi modô, Chrifte, tíi. all ding zwarzeitlich hier vergehn, nothroendig RönnaSie nicht beftehn. Denn ons nuirbleibt zú iederfrift Serbrauch deins Worts, fserr feth shrift.

Vue de Butzbach in der Wetterau (1625). La tour d'angle (a droite) montre le tube optique braqué vers 1" horizon (in Daniel Meisner, Eberhard Kieser, Thesaurus
Philopoliticus oder Polititsches Schatzkasstlein, Bd. 1, Buch 5, Nr.8, fac-similé del' 'édition de Frankfurt-am-Main, 1625-1626 et 1627-1631, Nördlinen, 1992).

Nouveaux matériaux pour l' histoire

de l' astronomie moderne : autour du landgraviat de

Hessen-Butzbach, 1609-1630

PAR PIERRE JEANDILLOU ET ÉDOUARD MEHL

$\grave{\mathrm{A}}$

la mort du landgrave Philippe Ir de Hesse « le Magnanime » (1504 1567), qui fut I' un des principaux soutiens politiques de Luther au temps de la Réforme, le vaste territoire sur quatre fils : la Hesse-Kassel échut à son premier fils Guillaume IV (1532-1592); la Hesse-Marbourg à so second fils Louis IV (1537-1604); la Hesse-Rheinfels a Philippe II (1541-1583), et la Hesse-Darmstadt Georges Ier (1547-1596). A la mort de Louis IV, en 1604 celui-ci n' ayant pas d' héritier, la Hesse-Marbourg fut partagee entre ses neveux, et Marbourg elle-même fut tattachee à la Hesse-Kassel, où le fils et successeur de Guillaume IV, Moritz von Hessen-Kassel (1572-1632), y imposa le calvinisme, tandis que les parties echues aux landgraves de Darmstadt demeuraient dans la confession luthérienne.

Sous le regard attentif du roi de France Henri IV ${ }^{1}$, une longue rivalité politique et confessionnelle entre Kassel et Darmstadt, attisee par le conflit genéralise de la guerre de Trente Ans (1618-1648), structure ce paysage politique morcelé et passablement instable, qui favorise néanmoins l' autonomie temporaire des plus petites subdivisions, comme le landgravia de Hessen-Butzbach, administré de manière indépendante par le landgrave Phillpe III (1581-1643). En , les landgraves de Darmstadt et Butzbach fonden - universite lathérienne de Gießen, pour contrecarrer infue grandissante de Kassel et de la réforme Iviniste imposée à l' universite de Marbourg.

\section{- - La bibliothèque du «liber naturae »}

Dès ses années de formation, Philippe de Hessen-Butzbach manifeste un intérêt précoce pour les questions scientifiques. Le landgrave fait partie du très petit nombre de ceux qui ont eu le privilège avoir des contacts épistolaires directs à la fois avec "excellentissime et doctissime Signor Galileo Galilei » et, un peu plus tard, avec le mathématicien impérial Johannes Kepler. Dès son accession au pouvoir, en 1609 quand commencent a circuler en Europe la lunette de Galilee et son Messager céleste annonçant en termes sobres et concis la découverte, sidérante et irrécusable, 
d' un nouvel horizon cosmologique ${ }^{3}$ - , Philippe développe la bibliothèque et fait faire des transformation dans le château pour y installer un observatoire astrohomique (1625, voir ill. p. 62). L' inventaire de cette bibliothèque aujourd' hui dispersée est d' un intérêt majeur pour 1 histoire des sciences à âge classique. Éabli tout au long de la guerre de Trente Ans par le mathématicien Daniel Mögling (1596-1635), dont le landgrave s est adjoint les services a partir de l' année 1621, I inventaire comporte, outre les livres répertorié selon leur discipline d' appartenance (arithmétique, géométrie, astronomie, astrologie, géographie, architecture, chimie, botanique $\cdots$ ), deux inventaires complementaires distincts : I un concerne les maquettes et modèles réduits (" Allerley Modelle», f. 13) ; l' autre, qui nous intéressera plus spécifiquement ici, concerne les instruments astronomiques d observation et de mesure (" Allerley Instrumenta », ff. 14-17) . L $^{2}$ observatoire de Butzbach fournit l' exemple paradigmatique d' une bibliothèque moderne, entierement, exclusivement presque religieusement consacrée au déchiffrement du "livre de la nature "

Bien que l' activité scientifique du landgrave et de son ingénieur d' études aient contribué de manière décisive, au dire de Kepler lui-même, au progrès de la recherche scientifique dans la première moitié du 17 siècle, cet apport est resté jusqu' à aujourd' hui largement méconnu. Cet oubli a une double explication. D' une part, Philippe III n' ayant pas de successeur, Butzbach a perdu son indépendance politico-administrative après sa mort, en 1643. Une partie de la bibliothèque de la "Reißkammer " "est revenue, via Marburg, à I' université de Gießen ; 1 autre a disparu sans laisser de traces. Mais une autre raison, moins évidente, peut expliquer cette disparition. Elle tient à la biographie et à la personnalité de celui qui joue ici un rôle déterminant . Daniel Mogling. Avant de rentrer comme en religion au service du landgrave, celui-ci a commis, sous des pseudonymes variés, de nombreux opuscules apologétiques en faveur de la Fraternité de la RoseCroix . Il est également très lié à un mathématicien à la réputation sulfureuse, Johann Faulhaber (15801635), dont les vaticinations apocalyptiques défraien la chronique et lui valent même, au cours de 1 année 1619, un procès à Ulm, oú il est sommé de justifier ses opinions hétérodoxes et de clarifier la teneur de ce que ses contemporains appellent, non sans ironie, sa «cabale de Faulhaber, aurait pu être sérieusement inquiété s' avait alors trouvé refuge à la cour de Butzbach et gardél anonymat dans les années qui suivirent. Kepler, qui le connait assez pour lui confier la garde de sa fille Suzanne dans les annees 1620, ne prononce jamais publiquement son nom, bien que Mögling fasse partie de ses plus zélés disciples et collaborateurs scientifiques. Premier témoin des travaux du mathématicien impéri concernant les logarithmes et leur usage dans le calcul astronomique, Mogling a fait la dénonstration de sa parfaite maitrise de ces nouvelles techniques calculatoires, dans des opuscules manuscrits aui ne sont jamais sortis de la bibliothèque personnelle du landgrave, et dont 1 impeccable serieux scientifique $n$ a plus rien a voir avec les fatras théosophiques de ses prenieres annés (voir ill. ci-contre) . La connaissance de ces travaux permettra de corrigerl avis des éditeur de Kepler, selon lesquels le nouveau calcul keplérien présenté dans les Tables rudolphines (1627) n' a eu aucun écho critique ni aucune réception significative en Allemagne ${ }^{10}$

\section{- - - - L'invention de l'observatoire moderne,}

\section{d'Uraniborg à Butzbach}

L' observatoire de Butzbach, bien que disposan un instrumentarium très complet et diversifié ${ }^{\prime \prime}, \mathrm{s}^{\prime}$ est spécialisé dans l' étude d' un phénomène détecté depuis le début de la décennie 1610 : les taches solarres. Dan une assez vaine querelle, Galilée avait revendique la priorite dans la découverte de ce phénomène quil suscite, aujourd hui encore, I intêrêt des astrophysiciens ${ }^{1-}$. Bien qu ils agisse d un phenomène local, dont la nature exacte était encore mal comprise, les taches solaires constituent, en tout ce qui concern 'astronomie et la cosmologie, le principal point d' entrée dans le débat scientifique de l' époque. Sous la houlette du landgrave, et à $\mathrm{l}$ ' aide $\mathrm{d}$ " un instrument unique en son genre, Mögling a recueilli des observations sur lesquelles, peu avant sa mort, Kepler s est appuyé pour soutenir sa derniere bataille copernicienne, propulsant de fait Butzbach au centre d' une discussion qui gagne rapidement une dimension internationale. $C^{\prime}$ est ainsi,

\section{$26+5$ \\ STBENA. A.TRONOMICA. \\ Brevisstmis 82 perspicuis Regue \\ C'alculum nosüm

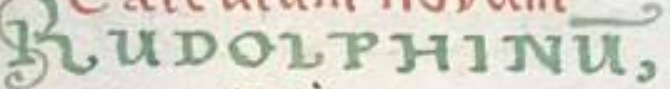 \\ Tam}

LUTMINARJUM

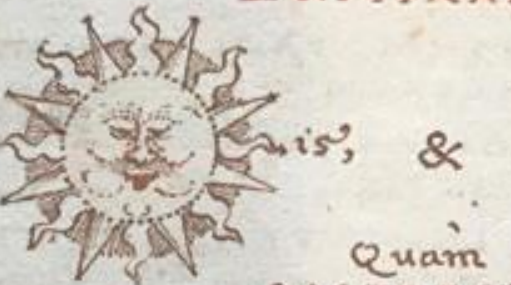

CлєT\& RоRUM

PI: A N F I A Rlim;

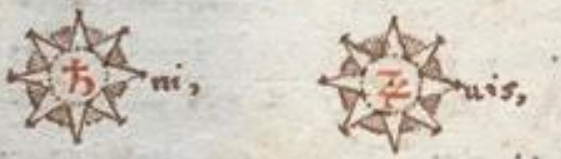

Inferiorumo six tom.

Una crim

DLI QUโ11 C

$$
\text { OBSER R }
$$$$
\text { moxo. }
$$

\section{Aftis

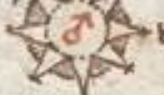
C, moDo.

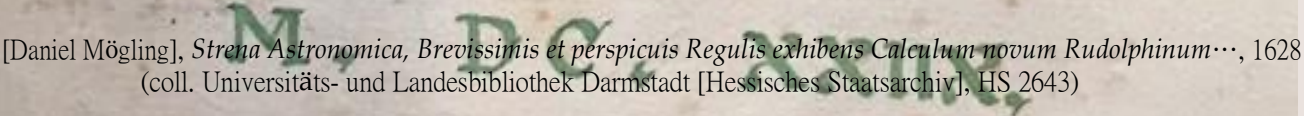




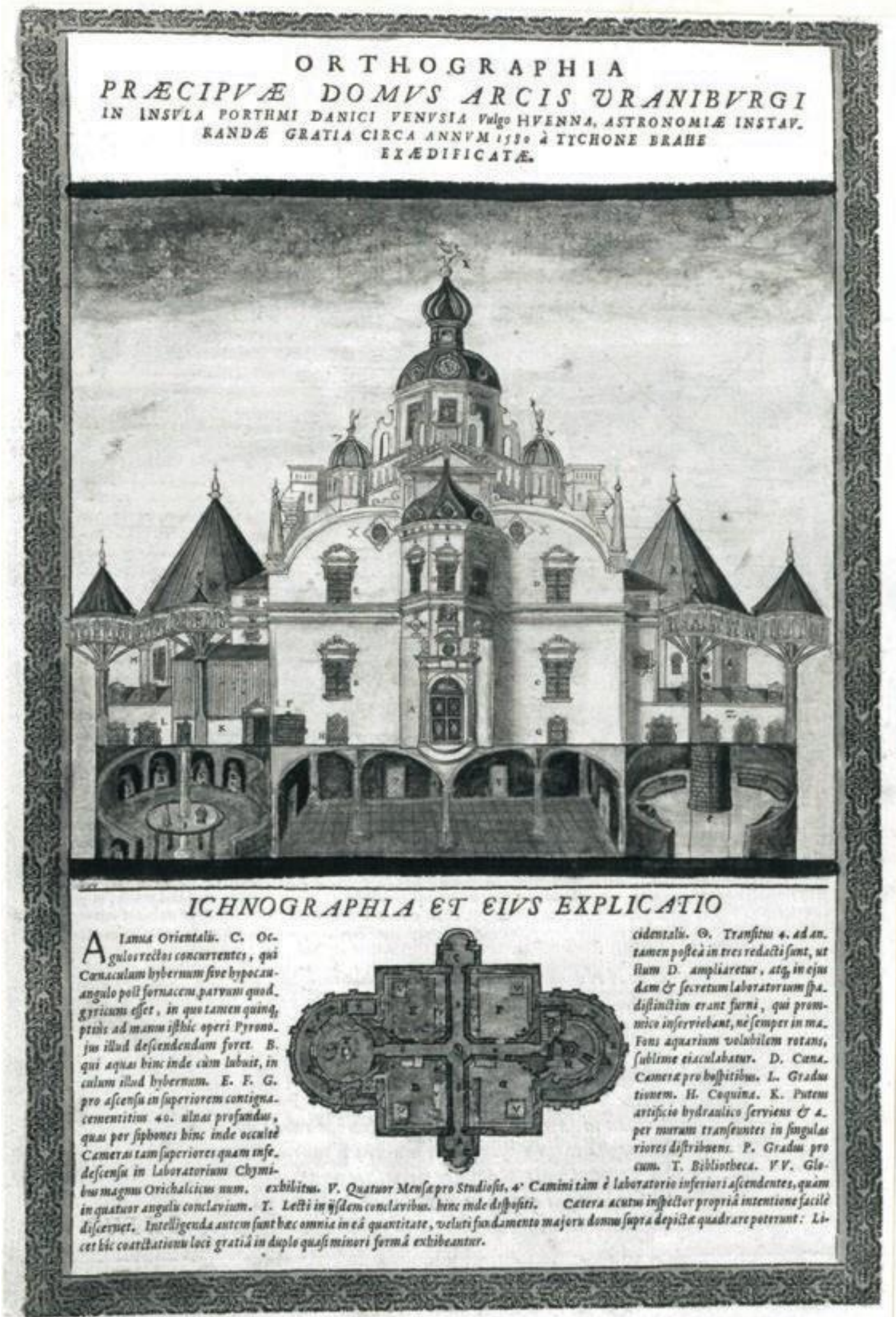

par une contribution aussi discrète que précise, que Butzbach éclipse pour un temps sa rivale Kassel, pourtant réputée dans toute l' Europe savante comme le fleuron de la recherche scientifique, depuis que échange mathématicien de Kassel, avec l' astronome danois Tycho Brahe (1546-1601) avait bouleversé le champ des études astronomiques dans la seconde moitié du $16^{\circ}$ siècle ${ }^{4}$. Unique en son genre, le conservatoire-observatoire de Butzbach n' est possible qu' en réunissant en un seul et même lieu le savoir théorique sur la construction des instruments, les movens financiers et les compétences techniques pour leur fabrication, sans oublier l' idée, toute neuve, selon laquelle une théorie scientifique, quelle qu' elle soit, n' a de validité et même de sens qu' à condition d' être sanctionnée par une expérience décisive révélant sa nécessité ${ }^{15}$. Mais, comme l' a bien noté quelques années plus tard l' auteur du Discours de la méthode, la production de telles expériences exige du temps et des moyens qui ne sont pas à la portée d' un simple particulier ${ }^{16}$. Tycho Brahe en avait donné un célèbre (contre-) exemple, persuadant son souverain Christian IV du Danemark de construire pour lui à grands frais l' invraisemblable observatoire d' Uraniborg sur l' île de Hven (voir ill. ci-contre). L' observatoire de Butzbach en est une imitation certes pâlotte, mais surtout beaucoup plus raisonnable

C' était là, au milieu d’ un bout de désert battu par les flots, que Tycho avait installé ses instruments d' observation d' une précision révolutionnaire, ainsi qu' un moulin à papier et une imprimerie destinés à l' impression de ses ouvrages. Le bâtiment, construit selon le plan dune architecture idéale, proposait toutes les commodités de l' époque moderne : chauffage central, eau courante, bibliothèque scientifique et espaces réservés aux chercheurs invités. Que rêver de plus ? Surgi comme par magie du génie un peu dérangé de Tycho Brahe, Uraniborg donnait un exemple, certes un peu démesuré et excessif, d' un dispositif complexe réunissant les conditions propices - et désormais indis- pensables - au progrès de la recherche scientifique.

Ce dispositif se trouve savamment et subtilement évoqué par le frontispice des Tabulae rudolphinae de Kepler (1627 ; voir ill. p. 69). L' histoire de ces nouvelles tables astronomiques est longue et complexe ; il suffira de retenir ici que le projet remonte à l' époque déjà ancienne où Kepler a travaillé auprès de Tycho, alors considéré, plus encore que Copernic, comme le rénovateur de l' astronomie à l'époque moderne. Pour diverses raisons, ces nouvelles Tables, tardivement parues, doivent donc célébrer la mémoire d’ un savant dont Kepler ne partage aucunement les vues, et même d un adversaire théorique qu' il était cependant obligé, pour des raisons strictement politiques, de reconnaitre, a son corps défendant, comme son mentor.

L' image $p .69$, d' une construction très sophisliquée, représente un tempietto aux dimensions tout à fait comparables à l' observatoire de Butzbach, au plafond duquel est représenté le système héliocentrique de Copernic ${ }^{17}$ Au centre, on voit Tycho Brahe interrogeant Copernic : « quid si sic ? » [et s' il en était ainsi ?] demande l' astronome danois en pointant l' index vers a Terre - qu' il prétend, contre Copernic, et a fortior contre Kepler, reposer immobile au centre du monde. Ce temple pourrait être un monument à la gloire de Tycho ; mais l' image est à double fond, et le lecteur attentif comprendra vite qu' il s' agit plutôt d' un tombeau de l' astronome danois. En effet, le tempietto aux dix colonnes est surmonté par un dôme sur lequel figurent six Muses. Chacune d' elles représente les nouveaux instruments et moyens techniques de l' astronomie keplérienne : l' une des Muses tient dans une main un aimant, et dans l' autre une aiguille ; une autre tient ce qui semble être une lunette de Galilée (mais pourrait etre aussi un tube optique fonctionnant comme une " camera obscura »); une troisième figure tient les logarithmes, que Kepler déduit d' une théorie générale des proportions ou rapports ; une autre encore tient une balance dont le soleil constitue le centre d' équilibre, et les planetes les poids - schématisant ainsi le principe fondamental d une nouvelle "physique céleste » keplérienne dont le noble et orgueilleux Danois $n$ ' avait pas même soupconné la possibilité. Autrement dit, le frontispice des Tabulae rudophinae montre, d' une manière assez subtile pour paraître élogieuse, la défaite de l' astronome danois incapable d' accéder aux vrais moyen d' observation et de calcul de l' astronomie moderne ! 


\section{- - - - Kepler et les taches solaire}

Le second séjour de Kepler à Butzbach a lieu en octobre 1627, immédiatement après qu il a amene lui-même, fin septembre, quelques exemplaires des Tabulae rudolphinae à la foire de Francfort. C' est à ce moment précis qu il prend connaissance des nouveaux instruments du landgrave, et des observations solaires menées par Daniel Mögling à l' aide d' un spectaculaire tube optique. Kepler a été le premier à publier une des- cription de cet instrument sans exemple ${ }^{18}$

"Une fois ces affaires réglées, je me sui rendu à Butzbach avec le très illustre landgrave Philippe de Hesse, et j ai pris le temps d' y contempler les instruments et exercices astronomiques du très estime Prince ; il y aurait beaucoup à écrire pour l' éloge de ces derniers, mais ce $n$ est pas ici mon propos. Afin cependant de ne pas priver les lecteurs de tout $l$ agrément des spectacles, $\mathrm{j}$ en evoquerai un seul, qui $\mathrm{n}$ a pas aissé de $\mathrm{m}$ émerveiller, quoique je sois particulièrement familier des observations. Dans un endroit dégagé et spacieux, on fixe un pieu d une trentaine de pieds de hauteur ; au sommet, on place une poulie ; par cette poulie, on fait passer un câble de cabestan qui entoure un tube immense de cinquante pieds, mû à grand peine par six hommes robustes depuis son fâte ; on élève ce tube à une hauteur telle que, par son trou - qui fait la taille $d$ ' un petit pois, ou bien d une lentille, ou même d' un grain de millet - le Soleil projette ses rayons sur une tablette blanche opposée, qui termine la cavité du tube à son extrémité. On distingue alors clairement, sur la tablette, les taches du Soleil, qui sont formées par le simple trou, sans l' interposition d' aucun verre convexe ; les figures, recueillies plusieurs jours d' affilée à midi, attestent que le mouvement des taches décrit une ligne qui est, aux solstices, perpendiculaire à ligne merridienne, et quil forme aux équinoxes un angle complémentaire à l' obliquité de l' écliptique, en changeantd hemisphered un equinoxea $\mathrm{l}$ autre in opposito equinoctio plagae etiam contrariae]; en sorte que cette ligne de mouvement se situe toujours dans le plan de écliptique. C est pourquoi, si la tache est fixe à la surface du soleil, il s' ensuit que, de la même manière que celle-là, le globe solaire lui-même, dis-je, $s$ avance en suivant 1 ecliptique ; nous en concluons ainsi qu' tourne autour de son axe et des pôles. Ajoute cet argu- ment à tous ceux que $\mathrm{j}^{3}$ ai déjà mentionnés dans l' Epit Ast. Lib. IV p. 514 num. 4, et demande-toi si quoi que p. 514 num. 4 , et denir pour toi s certaine proposition suivante : LE SOLEIL EST L' ORIGINE DU MOUVEMENT DES PLANETTES

Mögling n’ ayant jamais rien publié sur le sujet, les lecteurs devaient croire Kepler sur parole. Or les observations de Butzbach, tout récemment tirees l oubli où elles sommeillent depuis bientôt quatre cents ans, confirment entièrement les propos de l astronome imperial - à un détail près qui sera bientôt corrigé par ' astronome Wilhelm Schickard ${ }^{1}$. Le soin et 1 exactitude apportés par Mögling à l' observation des taches solaires fait du manuscrit HS 228 (voir ill. p. 7) un document d un intérêt inestimable pour l' histoire des sciences : non seulement comme temoignage de première importance sur la discipline émergente qu est la physique solaire, mais aussi, voire davantage encore, parce que la défense par Kepler de la valeur de ces observations menées de maniere quasi confidentielle a declenche, venant de étranger (Philipp Lansbergen, Martin Hortensius, Isaac Beeckman) une vague d' hostilité sans précédent, et une mise en cause des fondements mathematiques, optiques et observationnels de sa theorie. Cette attaque coordonnee vient des Provinces-Unies, et plus specialement de Middelburg, capitale de la province de Zélande et, pourrait-on dire, capitale du télescope, qu Hortensius et Beeckman préfèrent au tube optique pour l' observation des taches solaires. Or la discussion théorique qui oppose les Zélandais à Kepler sur les avantages respectifs du telescope à lentille et du simple tube optique s avere, en dépit de son apparence modeste et localiseee, d une très grande importance pour l' histoire de la science classique, puisque $i$ est des brumes de cette discussion qu' émergera bientôt, comme un soleil matinal, la Dioptrique de Descartes.

NOTES

Correspondancce inélite de Henri IV, roi de Franceet de Navarre, aver Renouard, 1840

Selon 1' adresse d' une lettre non datée, dont la minute est conservée au Hessisches Staatsarchiv U Universitiats-und Landesbibliothek pourl' aide qu' elles nous ont apportée dans I' exploration du fonds

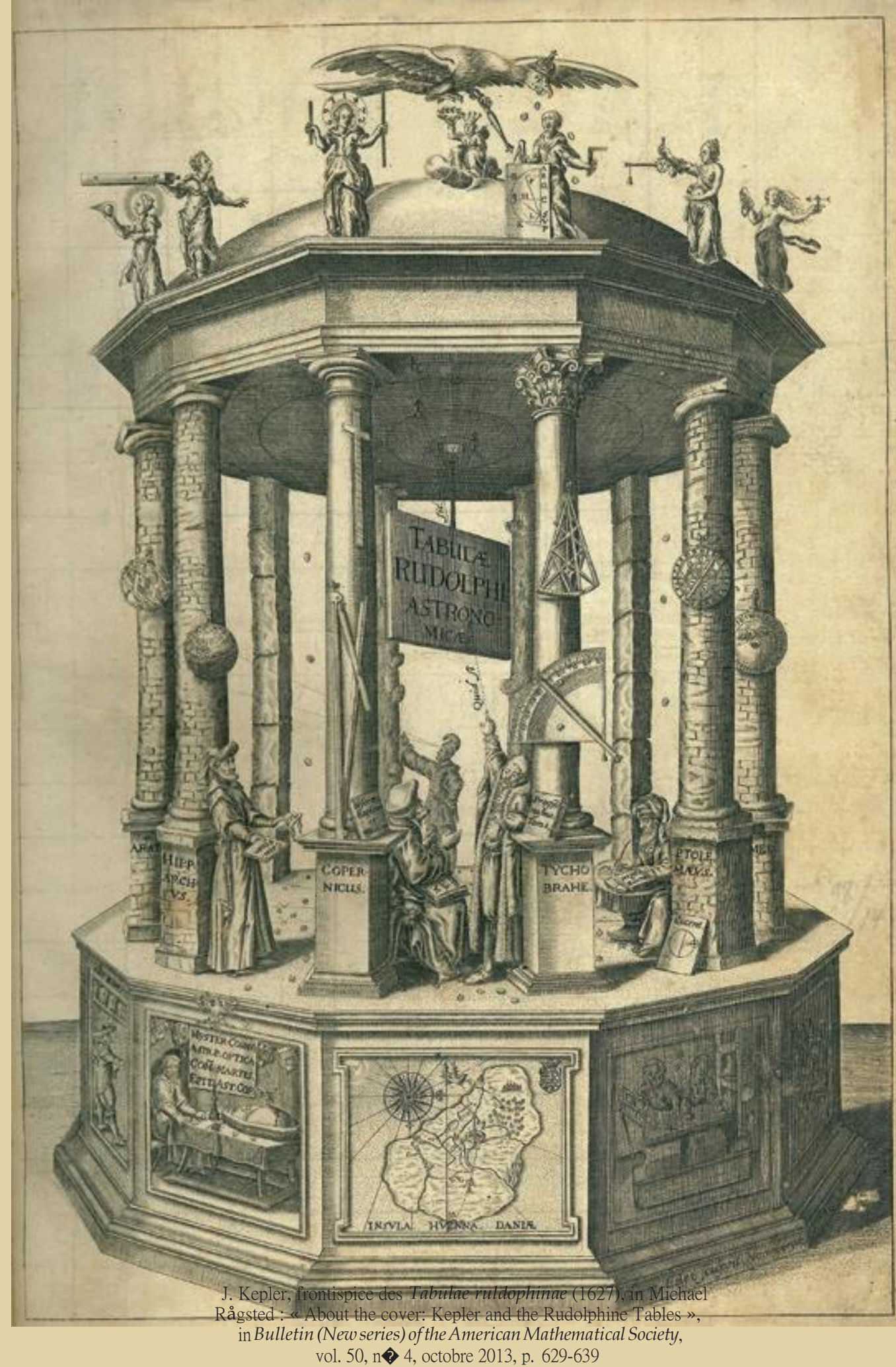

vol. 50, n 4, octobre 2013, p. 629-639 
3- Sur la diffusion du télescope, voir Massimo Bucciantini, Michele Cambrota, Franco Giuddce: G Galleleo' sTelescope. A european Story,
Cassachussetts), London, Harvard University Press, 2015. Surle Messager céleste (Sidereus nuncius) de Gailíée(1610), et Philippe Hamou, La mutation du visible. Essai sur la portée épistémologique desinstrumentsd'optique
Presses universitares du Septentrion, 1999.

- Inventar überdie Reißkammer Landgrav Philippsvon Butzbach, geschriebenvon Daniel Mögling im November 1628 mit Nachträ̈gen

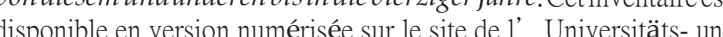

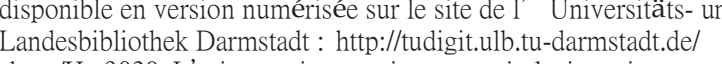

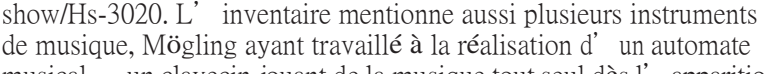
musical - un clavecin jouant de la musique tout seul dès 1' apparition

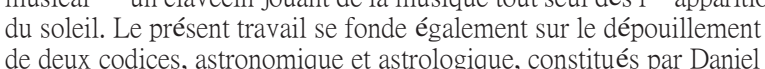
de deux codices, astronomique et astrologique, constitués par Danie
Mö́ling. HS 2608 et HS 2526 Le HS 2608 compotte notar un bref manuscrit astrologique de Kepler, édité dans le dernier

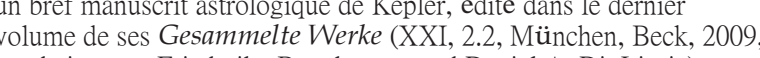
bearbeitet von Friederike Boockmann und Daniel A. Di Liscia). Malheureusement, une taute de plume dans le report d" une, date
a fait que le manuscrit keplérien a été mal contextualisé $:$ il n ${ }^{\prime}$ est pas arrivivé à Butzbach en 1612, comme $l^{\prime}$ affirme KGW XXI, 2.2, p. 507 et 633 , mais en 1621 , lorsque Kepler a fait son premier séjour traite dans ce texte est un horoscope pour une naissance datée

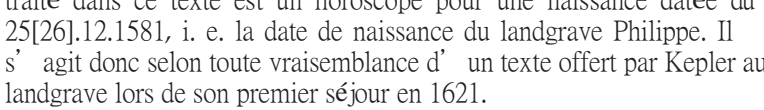

5- L' expression peutparaâtreexcessive, maiselleest, avec Kepler, « sacerdos libri naturae » (voir par ex. Kepler à Georg Herwart von Hohenburg, 26.3.1598, letrre nष $91, \mathrm{KGW} 13,193)$. Sur ce phénomène

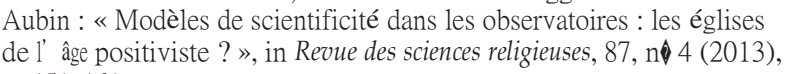

6- Ce terme, qu' on ne trouve dans aucun dictionnaire, mais qui
apparât dans certains inventairs dont cellui de la collection d’" art (Kunstkammer) de la cour de Dresse en 1587, semble venir d un des sens du verbe «reissen $\gg$ (dessiner). II dessignerait aninsi le lieu

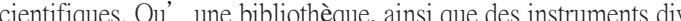
lui soient adjoints semble naturel pour la constitution de ce qu' on peut qualifier de «dispositif de savoir "

- Voir la notice de la Deutsche Biographie établie par Ulrich Neumann donne d' ailleurs pas une liste bibliographique complète des tra

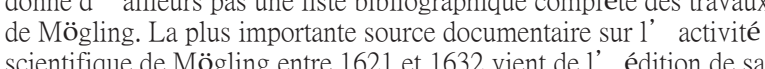

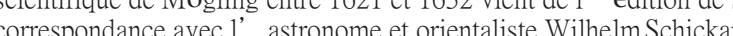

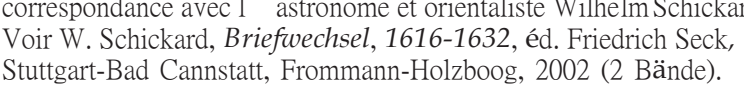

8- Sur cet épisode, voir Ivo SChneider, Johannes Faulhaber, 1580-
1635. Rechenmeister in einer Welt des Umbruchs, Brel Bo-

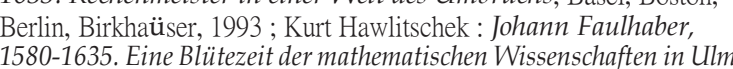
Ulm, Veröffentlichungen der Stadtbibliothek, 1995. Selon une hypothèse déjè ancienne, le jeune Descartes aurait pu assister à
ce procès : voir Lüder Gäbe, Descartes Sellbstkritik. Untersuchungen
zurPhilosophiedesjungen Descartes, Hamburg, Meiner, 1972. Cette hypoliese a servi den et augmentée. Édouard Mehl Descartesen Allemagne Lecor Ilemand del lélaboration de la science cartésienne, Strasbourg, Presses universitaries de Strasbourg, [2001], 2019.

-C C'est par ' 'entremise et à la demande du landgrave Philippe III

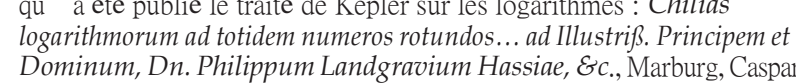

10, Nachbericht (Franz Hammer), 85

$1-\mathrm{L}$ ' inventaire (environ 180 entrées) mentionne plusieurs globes célestes, quadrants, sextants, compass éométrtiques, lunette de
Galiliee, cristaux pour étudier la diffraction et réfraction de la lumière, boussoles et instruments magnétiques pour 1 orientation et la mesure de la déćlinaison magnétique $\cdots$. La plupart de ces
instruments sont construits en laiton et non plus en bois, ce qui augmente considérablement leur précision, en même temps que leur
coût de fabrication. Voir Ernst Zinner, Deutsche und Niederländische 1965. L' unique description du grand globe céleste construit par
le landgrave entre 1621 et 1632 se trouve dans une des premieres sommes de muséologie répertoriant les cabinets de curiosités au (1657-1729): Museum Museorum.... [zweiter Teill. Frankfurt an Main, 1714, appendiX XVI, p. 57. On apprend dans ce seul et unique document que le globe representait le portrait du landgrave In corde
Leonis (Régulus a 2 Leonis end de sonépouse aul lieude Spica Viroinis (a Virginis) - ces deux étoiles formant avec Arcturus ( $\alpha$ Bootis un astérisme traditionnellement désigné comme le « triangle de

12- La dispute pour la primeur de cette découverte marque le début des hostilités entre Galilée et les jessuites, représentés par le pere Christoph Scheiner (1575-1650). En réalité, les taches ont été observees de maniliere incependante par David Fabicicus : voir M. Folkerts, «Johannes Kepler und David Fabricius », in Kepler.
La physique céleste. Autour del $l^{\prime}$ Astronomia nova (1609) éd Edouard La physique celeste. Aut Lour de l' Astronomian no
Mehl, Paris, Les Belles Lettres, 2011, p. 43-66.

13- Comme en temoigne 1 envol par la NASA de la sonde Parker (Parke Solar Probee en aoút 2018. Cette sonde a pour misssion d observer

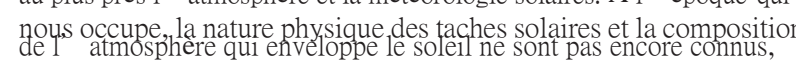
mais c' est justement Kepler qui a déduit, sur la base des observation
de Butzzach l' physiques et optiques spécifiques.

4- J. Bernoull cite une lettre inédite de Willebrord Snell au landgrave Moritz von Hessen-Kassel, exhortant celul-ci à remettre en route le programme des observations délaissé depuis la succession de son
père Guillaume IV ; cf. Jean Bernoulli, Lettres astronomiques où $I^{\prime}$ n donne une idée del'état actuel del'astronomie pratiquedans plusieurs villes de l'Europe (1771, p. 49). Surl' activité astronomique à Kassel auttour de Guillaume IV et du coperricien Christoph Rothmann, voir
Jürgen Hamel, Dieastronomischen Forschungen in Kassel unterWilhel IV (Acta Historica Astronomiae, vol. 2), Harri Deutsch Verlag,
2002; Christoph Rothmann'sDiscourseon the Cometof 1585 . An Edition and Translation, with Accompanying Essays, Miguel Á. Granada, Ada
Mosley \& Nicholas Jardine (éd.), Leiden-Boston, Brill, 2014 . - Voir à ce sujet 1' éloquente lettre du landgrave Philipp à Kepler, datée du mois de juin 1623 (lettre n $\$ 954, \mathrm{KGW} 18,130$ ) : les hypothèses
$l^{\prime}$ astronomie reposent sur deux pieds, à savoir les observations

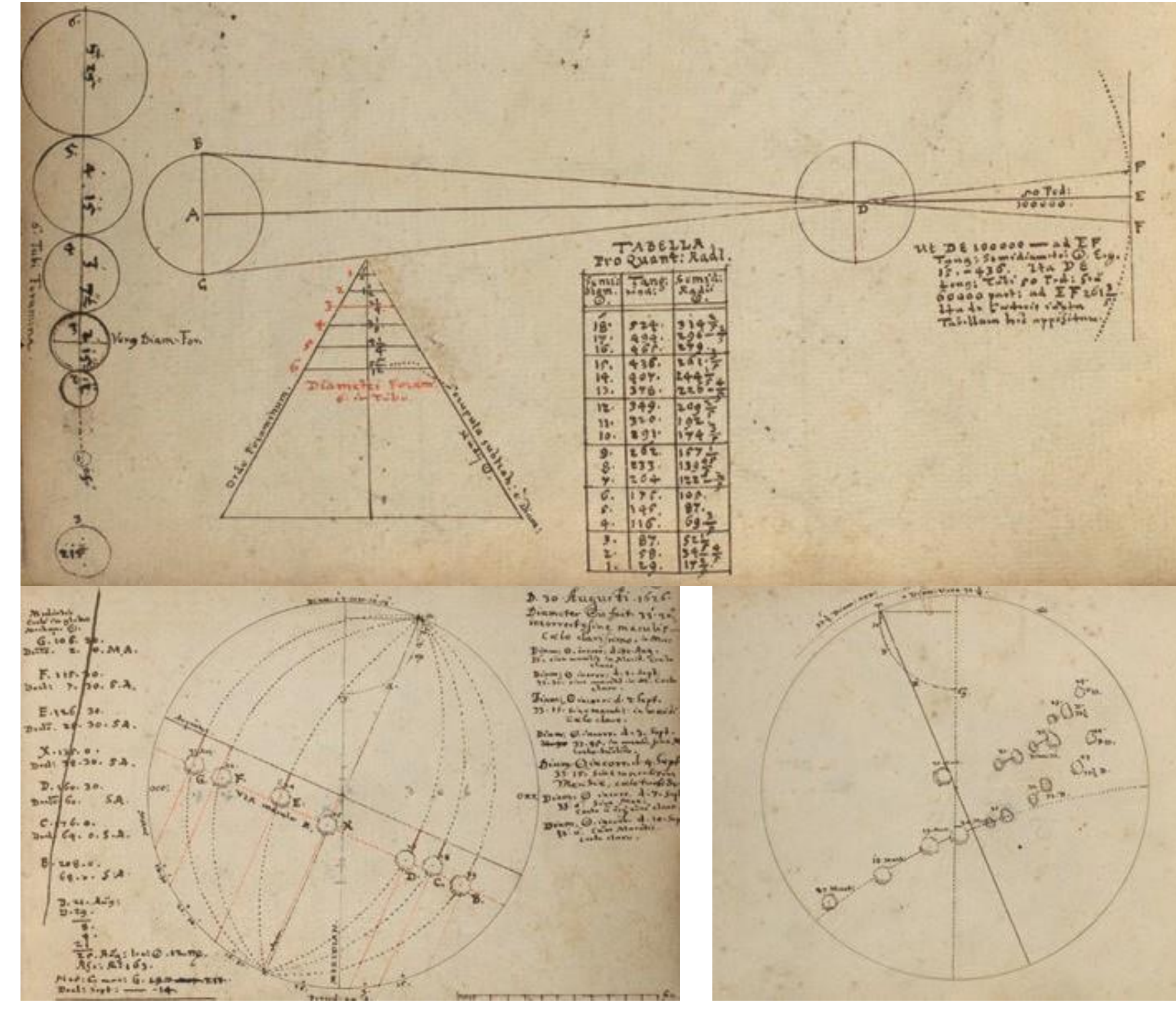

[Daniel Mögling], Observationes macularum solarium, 1626-1630

(coll. Universität- -

et le calcul. Pour les premières, il faut des instruments, mais le
landgrave évoque la difficulté de les tili iser avec la plus grande langrave évoque la difficulté de les tutiliser avec la plus grande
exactitude, sans que nes' yglissele risqued' erreurs. Pourle second, il faut des méthodes de calcul, et $c^{\prime}$ est pour cette raison que le landgrave demande à Kepler de lui fournir des tables logarithmiquus
destinees à abréger des calculs autrement tinterminables.

16- R. Descartes, Discours dela méthode pour bien conduire sa raison et trouver lavéritédanslessciences (1637), in CEuvres complètes sous la direction de Jean-Marie Beyssade et Denis Kambouchner, Paris,

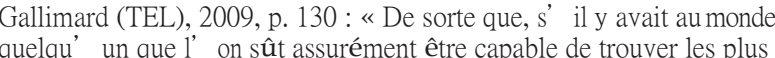
grandes choses et les plus utiles au public qui puissent être, et

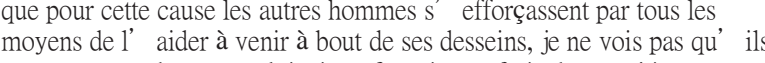
pussent autre chose pour livi sinon fournir aux frais des expésiences pussent autre chose pour lui, sinon fournirir aux frais des experrience
dont il aurarit besoin, et du reste empêcher que son loisir ne lui fût
ôté par l'
17- Sur le frontispice des Tabulae rudolphinae, voir Michael Rågsted:
«About the cover: Kepler and the Rudolphine Tables », in Bulletin (New series) of the American Mathematical Society, vol. 50 , n 4 , octobre 2013, p. 629-639.

8- Kepler, Ad Epistolam.... Jacobi Bartschii... Responsio : de computatione 19- Dans sa Pars responsiad epistolas P. Gassendi [...] de Mercurio sub sole Schickard nomme Daniel Mögling (ce que Kepler ne fait pas) et corrige la description de Kepler en ce qui concerne le diamètre de ouverture, ou « foramen ", par lequel la lamière entre dans le tube :
il n' $n^{\prime}$ est pas de la taille d" un grain de mill, comme le dit Kepler n' a probablement pas pu s' en approcher assez pour le constater de comme on le voit ci-dessus, le tube dispose de six diamè d" ouverture dont la taille varie de $1 / 2$ pouce à $3,5 \mathrm{~cm}$. Kepler a en fain
décrit la taille approximative des trois plus petits (" de la taille d" un décrit la taille approximative des trois plus petits ( «de la taille
petit pois, $d$ " une lentille, ou même $d^{d "}$ un grain de millet »). visu -, mais d"environ $1,5 \mathrm{~cm}$, soit la taille d "une olive. En réalite, 